\title{
BMJ Open Study protocol for a prospective cohort study identifying risk factors for sport injury in adolescent female football players: the Karolinska football Injury Cohort (KIC)
}

\author{
Ulrika Tranaeus (D) , ${ }^{1,2}$ Nathan Weiss (D) , ${ }^{2,3}$ Victor Lyberg, ${ }^{3}$ Martin Hagglund (D) , 4,5,6 \\ Markus Waldén (1D) , 5,6,7,8 Urban Johnson (D) , ${ }^{9}$ Martin Asker (D) , 2,3,10 \\ Eva Skillgate (1) ${ }^{2,3}$
}

To cite: Tranaeus U, Weiss $\mathrm{N}$, Lyberg V, et al. Study protocol for a prospective cohort study identifying risk factors for sport injury in adolescent female football players: the Karolinska football Injury Cohort (KIC). BMJ Open 2022;12:e055063. doi:10.1136/ bmjopen-2021-055063

- Prepublication history and additional supplemental material for this paper are available online. To view these files, please visit the journal online (http://dx.doi.org/10.1136/ bmjopen-2021-055063).

Received 29 July 2021 Accepted 17 December 2021

Check for updates

(C) Author(s) (or their employer(s)) 2022. Re-use permitted under CC BY. Published by BMJ.

For numbered affiliations see end of article.

Correspondence to

Dr Ulrika Tranaeus;

ulrika.tranaeus@gih.se

\section{ABSTRACT}

Introduction Football is a popular sport among young females worldwide, but studies concerning injuries in female players are scarce compared with male players. The aim of this study is to identify risk factors for injury in adolescent female football players.

Methods and analysis The Karolinska football Injury Cohort (KIC) is an ongoing longitudinal study that will include approximately 400 female football academy players 12-19 years old in Sweden. A detailed questionnaire regarding demographics, health status, lifestyle, stress, socioeconomic factors, psychosocial factors and various football-related factors are completed at baseline and after 1 year. Clinical tests measuring strength, mobility, neuromuscular control of the lower extremity, trunk and neck are carried out at baseline. Players are followed prospectively with weekly emails regarding exposure to football and other physical activity, health issues (such as stress, recovery, etc), pain, performance and injuries via the Oslo Sports Trauma Research Center Overuse Injury Questionnaire (OSTRC-0). Players who report a substantial injury in the OSTRC-0, that is, not being able to participate in football activities, or have reduced their training volume performance to a moderate or major degree, are contacted for full injury documentation. In addition to player data, academy coaches also complete a baseline questionnaire regarding coach experience and education.

Ethics and dissemination The study was approved by the Regional Ethical Review Authority at Karolinska Institutet, Stockholm, Sweden (2016/1251-31/4). All participating players and their legal guardians give their written informed consent. The study will be reported in accordance with the Strengthening the Reporting of Observational studies in Epidemiology. The results will be published in peer-reviewed academic journals and disseminated to the Swedish football movement through stakeholders and media.

\section{Strengths and limitations of this study}

- A strength is the bio-psychosocial and multiprofessional perspective of the risk of injuries in young female football players and factors of importance for not being injured, even though the biopsychosocial factors are not equal included.

- Strengths are also the large sample size and the robust data collection of exposures, potential confounding factors, potential effect measure modifiers and outcomes.

- A potential limitation is the risk of misclassification of time varying exposures and outcomes in the weekly self-reported data collection.

- Using emails and SMS for weekly reports might decrease the response rates and thereby increase the risk of selection bias in the results. If the response turns out to be low, there is a risk of selection bias in the risk analyses.

\section{INTRODUCTION}

Four million females worldwide are registered football players, of which 2.5 million are under 17 years old according to Fédération Internationale de Football Association. ${ }^{1}$ Studies regarding injuries in female football players are fewer compared with the number of studies in male football players. ${ }^{2-4}$ In brief, these studies show that common injuries in female football players are joint and ligament injuries to the knee and ankle joints as well as muscle and tendon injuries of the thigh. In addition, there is a particular concern for concussions and anterior cruciate ligament injuries in female players. ${ }^{35-8}$

Female football players have more absence days from football due to injuries compared with male players, ${ }^{8}$ and long-term consequences of injuries might be considerable 
for young football players. ${ }^{9}$ For players with a history of injury, the risk of osteoarthritis in lower extremity joints are high and greater than in the general population. ${ }^{1011}$ Injuries may also lead to premature career ending, ${ }^{12}$ and mental health problems. ${ }^{13}$ Identifying risk factors for injury is, therefore, an important step towards reduction of injury risk. ${ }^{14}$ To identify possible risk factors, well-designed prospective cohort studies are needed. ${ }^{1516}$ Specifically, the suggested risk factors in this setting can be classified as bio-psychosocial factors (see Wiese-Bjornstal for bio-psychosocial view on a sport injury risk profile). ${ }^{17}$ Biological risk factors for injury in female players are previous injury, ${ }^{78-20}$ a hamstring/quadriceps ratio of less than $55 \%$, increased body mass index, as well as results of plyometric tests, for example, poor performance in drop jump landing test is associated with increased risk of ankle injury. ${ }^{21}$ Other biological risk factors associated with an increased risk of injury during the season are young age, ${ }^{618}$ physical complaints at the beginning of the season, ${ }^{18}$ familial disposition such as a parent/sibling ${ }^{18}$ or a twin ${ }^{22}$ with knee injury, and lower level of preseason aerobic fitness. ${ }^{23}{ }^{24}$ Findings regarding joint hypermobility as a risk factor in female players are inconclusive in older studies, ${ }^{2325}$ although no association was shown in more recently published studies. ${ }^{26}{ }^{27}$ Risk factors for back pain in adolescents include rapid growth rate, and tight muscle imbalance, ${ }^{28}$ but risk factors for football related back/neck injuries in young females are not known. Psychological risk factors reported includes somatic trait anxiety, mistrust, ineffective coping, ${ }^{29}$ life event stress ${ }^{30}$ and perceived mastery climate. ${ }^{20}$ Social factors that influenced the risk for injury in female athletes are coaches' and player's education regarding injury prevention strategies, ${ }^{31}$ stress from teammates and coaches, ${ }^{20} 2932$ and for back pain in adolescents; smoking. ${ }^{28}$ In football, an identified situational specific risk factor is the playing positions defenders and strikers. ${ }^{19}$

In summary, most knowledge about risk factors for injuries in adolescent female football players consists of isolated factors, and lack of using multidisciplinary and bio-psychosocial perspectives. Hence, the overall aim of the Karolinska football Injury Cohort study (KIC) is to identify risk factors for injuries in adolescent female football players from a bio-psychosocial perspective. Specific aims are to determine the incidence of injuries in young female football players and to identify modifiable risk factors for such injuries. Finally, our aims include to describe changes in muscle strength and range of motion (ROM) over a year, trajectories of pain and to identify important factors for not being injured over a year.

\section{METHODS AND ANALYSIS}

This is a prospective observational cohort study designed in agreement with Strengthening the Reporting of Observational studies in Epidemiology (STROBE) guidelines. ${ }^{33}$

\section{Study setting and participants}

Football clubs with adolescent female academy players aged 12-19 years, participating in Swedish divisions 1-2 for girls in the largest regions, are eligible to participate in the study. Most players will be recruited in Stockholm. The district of Stockholm consists of 140 teams and approximate 2520 female players, 13-19 years old. Clubs which meet the inclusion criteria are contacted and invited to participate and are given oral and written information. Clubs which choose to take part in the study are provided with a more detailed oral and written information in the presence of players, legal guardians and coaches.

A cohort of approximately 400 adolescent academy players will be recruited. An internal pilot study of 63 football players has been conducted to test the infrastructure and the implementation of the study, with satisfactory results (unpublished data).

\section{Baseline measurements \\ Questionnaires}

The baseline questionnaire covers potential risk factors for the aetiology of sport injuries as well as information about players' general health status. Players are surveyed in various areas, including health: health problems (eg, illness), medication, age at menarche, amenorrhoea; lifestyle: sleep patterns, eating habits, food supplements, tobacco as smoking or Swedish snus (snuff) and alcohol; and socioeconomic factors: guardians' education. Included football-related factors are: training and match play exposure, playing position, dominant limb, years of experience, other sports participation, injury preventive strategies (eg, the Swedish injury prevention warmup programme Knee Control) ${ }^{34}$ and type of turf at the home facilities (artificial or natural grass) according to guidelines for football studies ${ }^{35-37}$ Psychosocial factors are surveyed using: the modified General Health Questionnaire-12 consisting of 12 items regarding self-reported general psychological health using a four-point Likert scale, ${ }^{38}$ coping assessed by a 28 item self-report questionnaire that measure effective and ineffective strategies to cope with stressful events using a four-point Likert scale (Brief COPE) ${ }^{39}$ player's passion to sport measured in harmonious and obsessive passions using a 14 item questionnaire with a sevenpoint Likert scale (Passion scale), ${ }^{40}$ education in sport psychology, regularly seeing a sport psychologist/mental coach and perceived stress (single item question). ${ }^{41}$ Injury history: injuries occurring within the previous 6 months prior to inclusion are captured using a modified Swedish version of the validated psychometric instrument Oslo Sports Trauma Research Center Overuse Injury Questionnaire (OSTRC-O) ${ }^{42}{ }^{43}$ Back and neck pain is covering the frequency, intensity, disability of low back pain (LBP) and upper back pain/neck pain (UBNP) and corresponding longitudinal trajectories the preceding 6 months using modified versions of the Chronic Pain Questionnaire ${ }^{44}$ and Visual Trajectories Questionnaire-Pain, ${ }^{45}$ respectively.

In addition, coaches in the included teams are surveyed regarding their education, years of experience, the use 
of warmup and stretching regime and implementation of injury prevention programmes.

\section{Physical test protocol}

The physical test protocol includes several tests that are considered valid, reliable, and field friendly; performed in approximately $60 \mathrm{~min} /$ player. The protocol comprises measurements of strength, mobility and control of lower extremity, trunk and neck and also include anthropometric measurements (height, weight and leg length). The protocol is briefly outlined below, however, further details including visual presentations is available in the electronic supplementary file (online supplemental file 1 ).

All test procedures are conducted in indoors facilities during weekends. The physical tests are divided into nine test stations with 1-2 test leaders each (online supplemental file 1). Hitherto, 52 clinically experienced test leaders have been involved in data collection. They were trained by MA, VL, NW and the previous test leader in charge of the station to ensure consistent execution and reliability. Information and instructions given to the players regarding the tests are standardised, and test leaders refrain from coaching or encouraging the players in any way during the procedures.

A maximum of nine players are tested per session (ie, one at each station) and are informed to train and compete as usual prior to testing. Players are informed to refrain from certain tests that evoke pain, provoke ongoing injuries or other health-related issues. Prior to performing the physical tests, players complete a standardised $7 \mathrm{~min}$ warm-up programme comprising $4 \mathrm{~min}$ of jogging, $10 \times 1$ body weight squats, $10 \times 1$ body weight squat jumps and $10 \times 1$ unilateral body weight lunges. Following the warm-up session, players are randomly assigned to a starting test station and subsequently follow a predefined order.

\section{Calf heel raises}

Ankle plantarflexion (PF) muscle endurance is investigated using unilateral weight bearing calf heel raises. ${ }^{46}$ The player is instructed to perform maximum unilateral barefoot heel raises continuously to failure, guided by a metronome to standardise the pace $(1 \mathrm{~s}$ concentric, $1 \mathrm{~s}$ eccentric contraction). The test leader registers the number of accomplished repetitions and discontinues when the player fails to reach the marked target height. The same procedure is then conducted on the opposite foot.

\section{Active PF mobility}

Active PF ROM is measured with a universal goniometer in supine position using fibula and fifth metatarsal as reference marks. ${ }^{47} 48$ The player is instructed to maintain extended knees throughout the movement, and to perform a sequence of six maximal active PF cycles from a neutral dorsiflexion (DF) position, of which the final three trials are registered.
Weight bearing ankle DF mobility

Weight bearing ankle DF ROM is measured in a lunge position with the player's foot placed on a metric ruler $10 \mathrm{~cm}$ away from a wall. ${ }^{46}$ The player is instructed to lunge forward, until contact with the wall is achieved without allowing the heel to lift off the ground. Three warm-up trials are performed from the $10 \mathrm{~cm}$ mark to familiarise the player with the test. Thereafter, the test leader measures the following three trials. From the $10 \mathrm{~cm}$ reference mark, the player progresses $1 \mathrm{~cm}$ away at a time from the wall until unable to perform a successful repetition. If unable to perform a successful repetition at the $10 \mathrm{~cm}$ reference mark, she is asked to progress $1 \mathrm{~cm}$ forward until able to complete a successful repetition. The maximal DF ROM is measured with a digital inclinometer (Clinometer, Plaincode, Stephanskirchen, Germany) and distance from the wall to the greater toe is measured in centim.

\section{Trunk mobility}

Trunk rotation mobility is measured in a modified seated rotation test, and in a lunge position on a gym mat graded with $5^{\circ}$ increments. ${ }^{50-52}$ The player is instructed to maximally rotate alternating between right and left, in a crosslegged position and subsequently in a lunge position on the dominant, and non-dominant limb while the test leader measures the rotational degrees in the end range. Three repetitions are performed in each direction during the three separate positions, and the mean value for each position is later used for analysis.

\section{Trunk strength}

Isometric trunk rotational strength is measured in a modified standing wood chopper test using a force gauge to evaluate force output (RS Pro Digital Force Gauge, RS Components, Corby, UK).$^{53-55}$ In this modified test, the player holds a handle attached to the force gauge in shoulder height in a standing position. The player is instructed to generate force through her trunk and rotate for $5 \mathrm{~s}$ while maintaining straight arms. Three consecutive repetitions are conducted in each direction and the maximal force output is later used for analysis.

\section{Deep neck flexor endurance}

Deep neck flexor muscle endurance is assessed through a modified version of the cranio-cervical flexion test with a pressure sensor (Stabilizer Pressure Bio-Feedback, Chattanooga Group, Hixon, Tennessee, USA). ${ }^{51} 5657$ The test consists of a pretest and an endurance test. In the pretest the player is positioned in a supine position on an examination table and are instructed to slightly push the neck against the pressure sensor to increase the pressure and then maintain the pressure for $3 \times 3 \mathrm{~s}$, with a $3 \mathrm{~s}$ rest in between each contraction, at a specific target pressure (TP), starting at $20 \mathrm{~mm} \mathrm{Hg}$. If the player can perform this task, she is instructed to increase the pressure to $24 \mathrm{~mm} \mathrm{Hg}$ and keep the pressure for another $3 \times 3 \mathrm{~s}$. This is repeated with a $2 \mathrm{~mm} \mathrm{Hg}$ increase until the 
player reaches $30 \mathrm{~mm} \mathrm{Hg}$. If the player can perform the pretest the endurance test is subsequently performed. During the endurance test, the same setup and procedure as in the pretest is carried out. However, the player is instructed to hold each contraction at the TP for $3 \times 10$ $\mathrm{s}$ with a $10 \mathrm{~s}$ rest in between contractions. The highest completed TP with a full set of $3 \times 10 \mathrm{~s}$ contractions is later used for analysis.

\section{Hip and knee strength}

Isometric hip flexion, extension, adduction and abduction strength as well as eccentric hip abduction and adduction strength are measured with a hand-held dynamometer (HHD) (MicroFet2, Hoggan Health Industries inc. West Jordan, Utah, USA) ${ }^{5859}$ Furthermore, isometric knee extension strength is measured with an HHD and the player in a seated position with the knee joint in $90^{\circ}$ of flexion. Prior to executing the strength tests, two submaximal isometric contractions in each direction are performed to familiarise the player with the procedures. Three isometric contractions with gradually increasing power output for $5 \mathrm{~s}$, and three maximally eccentric contractions for $3 \mathrm{~s}$ are performed in the isometric and eccentric tests, respectively, with a 10 s rest in between contractions. The maximal power output for each position is later used for analysis.

\section{Hip mobility}

Measures of passive hip ROM in flexion and abduction in prone position and extension, internal and external rotation in supine position is obtained using a universal goniometer. ${ }^{6061}$ Three consecutive measurements for each position are performed for both the dominant and the non-dominant leg, and the mean value for each position is later used for analysis.

\section{Functional performance tests}

To assess the player's unilateral jump performance, the One-leg Long Box Jump Test (OLLBJ) and square hop test are performed. ${ }^{62} 63 \mathrm{~A} 40 \times 40 \mathrm{~cm}$ square is marked on the foundation and later used as a reference mark in both tests.

In the OLLBJ, the starting position are calculated by dividing the player's height $(\mathrm{cm})$ with 1.6 (height/1.6). Thereafter, the player is instructed to stand on one leg on the starting position and then jump on one leg directed inside the boundaries of the square and maintain balance after landing. Three warm-up trials and five consecutive test trials are performed on each leg. The total number of approved trials are registered by the test leader.

During the square hop test, previously described in detail ${ }^{62}{ }^{63}$ the player is instructed to jump on one leg in and out of the square as many times as possible for $15 \mathrm{~s}$ in a clockwise direction, timed with a stopwatch while the test leader registers the number of approved jumps. The player performs two warm up trials on each foot prior to executing the test.

\section{Ankle and knee stability}

To assess stability of player's talocrural joints, a modified anterior drawer test is employed. ${ }^{6465}$ Furthermore, a modified version of Fairbank's apprehension test is used to evaluate the player's stability in the patellofemoral joint. ${ }^{66}$ The tests are conducted on both the dominant and non-dominant foot and knee and are considered positive if the player experience any pain or discomfort during the examination, and/or an involuntary contraction of the quadriceps musculature occur during the Fairbank's apprehension test.

\section{Isometric back extensor endurance}

Isometric back extensor endurance is assessed by the modified Sorensen test. ${ }^{67-69}$ In this previously described modified test, ${ }^{6768}$ the player's lower body is supported by an examination table in prone position with three straps and the anterior-superior iliac spine is aligned with the edge of the table. The player is instructed to keep her arms folded across the chest throughout the procedure and isometric maintaining the upper body in a horizontal position until failure. The test leader registers the time elapsed until failure. A digital inclinometer (Clinometer, Plaincode, Stephanskirchen, Germany) is placed on a metric ruler at the level of the 5 th vertebra in the thoracic spine to monitor sagittal plane movement. Prior to the assessment, the player completes a shorter warmup trial to orient the desired sagittal plane target angle.

\section{Follow-up measurement and outcome}

Follow-up measurements are collected prospectively during 1 year from the baseline. In the weekly online questionnaire, the players are asked to answer questions regarding new and ongoing injuries, LBP and UBNP intensity, social support, perceived stress, recovery, and to be able to consider workload, number of training and match play hours/week. ${ }^{70}$ To assess whether players sustain football related injuries throughout the follow-up period, the Swedish version of OSTRC-O is employed and included in the weekly online questionnaire. ${ }^{42} 4371$ Two study specific adaptions were made to the OSTRC-O. First, a question regarding absence/reduced participation in training/match due to reasons not related to injuries was added. Second, the option to specify injuries in different anatomical localisations in the lower and upper extremity, back, neck, head and abdomen was included.

Football related injuries reported with the OSTRC-O in the weekly online questionnaire leading to moderate or severe reductions in participationand/ or sports performance or complete inability to participate in sport are classified as a substantial injury in this study. ${ }^{42}$ Players reporting new substantial injuries are contacted via telephone by a clinically experienced research assistant to answer a standardised interview with questions concerning the injury such as: injury mechanism, localisation, type, time-loss, re-injury, diagnosis and medical care. Injuries are divided into acute and gradual onset. An acute injury is defined as a result from a specific, 
identifiable event, whereas injuries with gradual onset are defined as an injury without a single, identifiable event responsible for the injury. ${ }^{35}$ Players receive an automated link to the online questionnaire sent by email each Sunday, with a reminder email the next day to players not responding. Furthermore, if no response is received, a text message reminder with the link is sent on Tuesdays. Finally, every other week representatives of the study visit participating football clubs to collect unanswered surveys for the previous 2-week period.

After 52 weeks of participation, a questionnaire with equivalent content as the baseline questionnaire (excluding OSTRC-O with 2 months and 3-6months recall) are distributed to the players to evaluate possible changes from the baseline characteristics. The first 106 included players also underwent a secondary physical test protocol after 52 weeks of follow-up. In the 1-year follow-up questionnaire, different aspects of UBP and LBP, respectively, in the preceding 6 months are measured. 'Have you had UBP/Have you had LBP' (Yes/No)? If yes, has the pain hindered your daily activities (No, Yes to some extent or Yes to a high degree)? If Yes, the 'VTQ-P' is used to capture the longitudinal state of a player's pain experience of UBP and LBP and are retrospectively reported for the preceding 6 -month period. ${ }^{45}$ See table 1 for an overview of the measurements during the different phases of the study.

\section{Sample size}

The statistical power for the analyses will depend on the exact research question, the number of exposed players and whether the exposure is continuous or categorised. The sample size in the KIC project is based on the definition 'a substantial injury' as proposed by Clarsen et $a t^{42}$ and back injuries in adolescent female players in a previously published study. ${ }^{7}$ Based on a relative risk of 1.9 for a substantial injury in the back/neck, when 88 of the players are exposed, and with a power of 0.80 , a significance level $5 \%$ and with potential $10 \%$ drop out and a follow-up time of 1 year to identify risk factors, 420 players will be included.

\section{Table 1 Summary of the included measurements during the different phases of the study}

\begin{tabular}{|c|c|c|}
\hline Phase & Measurements & Tests/tools \\
\hline \multirow[t]{6}{*}{$\begin{array}{l}\text { Baseline: players (consecutive } \\
\text { during inclusion; 2016-ongoing) }\end{array}$} & $\begin{array}{l}\text { Demographic information, general health status (history of pain, } \\
\text { illness, medication, plagues, menstrual cycle, back and neck } \\
\text { pain), lifestyle (sleep patterns, resilience, food supplements, use } \\
\text { of tobacco or alcohol), stress, socioeconomic factors (guardians' } \\
\text { education), football related factors (position, years of experience, } \\
\text { injury preventive strategies) }\end{array}$ & $\begin{array}{l}\text { KIC Baseline players, } \\
\text { The Chronic Pain } \\
\text { Questionnaire (CPQ), } \\
\text { Visual Trajectories } \\
\text { Questionnaire-Pain } \\
(\text { VTQ-P) }\end{array}$ \\
\hline & $\begin{array}{l}\text { Anthropometric measurements (height, weight, leg length), and } \\
\text { measurement of strength, mobility and control of lower extremity, } \\
\text { trunk, and neck }\end{array}$ & $\mathrm{KIC}$ test protocol \\
\hline & History of injury and complaints & Modified OSTRC-O ${ }^{42} 43$ \\
\hline & Passion & Passion scale ${ }^{40}$ \\
\hline & General health & $\mathrm{GHQ}-12^{38}$ \\
\hline & Coping strategies & Brief COPE ${ }^{39}$ \\
\hline $\begin{array}{l}\text { Baseline: coaches (consecutive } \\
\text { during inclusion; 2016-ongoing) }\end{array}$ & $\begin{array}{l}\text { Education, years of experience, the use of warmup and stretching } \\
\text { regime and implementation of injury prevention programmes }\end{array}$ & KIC Baseline coaches \\
\hline \multirow{4}{*}{$\begin{array}{l}\text { Weekly follow-up: players } \\
\text { (September 2016-ongoing) }\end{array}$} & Exposure to football training and match play & KIC weekly report \\
\hline & Exposure to other physical activity & \\
\hline & Health (eg, stress, recovery) and social support & \\
\hline & Report on pain, injury performance, complaints & Modified OSTRC-O ${ }^{42} 43$ \\
\hline $\begin{array}{l}\text { In case of a substantial injury } \\
\text { event }\end{array}$ & $\begin{array}{l}\text { Report on injury/complaint (type of injury, localisation, inciting } \\
\text { event) }\end{array}$ & $\mathrm{KIC}$ medical report \\
\hline $\begin{array}{l}\text { One-year follow-up: players } \\
\text { (consecutive after } 52 \text { weeks } \\
\text { participation: } 2017 \text {-ongoing) }\end{array}$ & $\begin{array}{l}\text { Football related factors (position, injury preventive strategies). } \\
\text { Health status (pain in back or neck) lifestyle (sleep patterns, } \\
\text { resilience, food supplements, use of tobacco or alcohol, physical } \\
\text { activity), stress, coping and passion for sport }\end{array}$ & $\begin{array}{l}\text { KIC 1-year } \\
\text { questionnaire }\end{array}$ \\
\hline $\begin{array}{l}\text { One-year follow-up (consecutive } \\
\text { after } 52 \text { weeks participation in } \\
\text { the first } 106 \text { included players) }\end{array}$ & $\begin{array}{l}\text { Anthropometric measurements (height, weight, leg length), and } \\
\text { measurement of strength, mobility and control of lower extremity, } \\
\text { trunk and neck }\end{array}$ & $\mathrm{KIC}$ test protocol \\
\hline
\end{tabular}

GHQ-12, General Health Questionnaire-12; KIC, Karolinska football Injury Cohort; OSTRC-O, Oslo Sports Trauma Research Center Overuse Injury Questionnaire. 


\section{Statistical methods}

The data in the KIC study will be used to answer several different research questions and therefore, different analyses methods and statistics will be used. Kaplan-Meier estimates will be used to describe incidence. Cox regression analyses or discrete time survival analyses will be used to measure the associations between exposure and outcome, and to adjust for confounding. Only players without substantial injuries the two preceding months (reported in the baseline questionnaire) will be considered in the risk analyses, and stratified analyses to examine effect measure modification will be performed when relevant. The development of injuries is likely complex. This justifies why we measure an extensive number of factors so that we can consider confounders, intermediators and effect measure modifier in these analyses. When identifying trajectories of time, and various factors generalised estimating equations will be used for these analyses to consider the covariance between repeated measurements.

\section{Time plan}

Players will be recruited from 2016 and followed weekly for 1 year regarding injuries/complaints. Players will consecutively be invited and included from the year they turn 13 years old and play in a participating club. The inclusion of participants will continue until we reach over 400 players.

\section{Data statement}

The dataset and statistical codes will be available on reasonable request when the data collection is completed.

\section{Patient and public involvement}

No patient involved.

\section{ETHICS AND DISSEMINATION}

The study was approved by the Regional Ethical Review Authority at Karolinska Institutet, Stockholm, Sweden (2016/1251-31/4). All participating players and their legal guardians receive written and oral information regarding the study and give their written informed consent when entering the study. Players under the age of 15 are required to have written informed consent from their legal guardians. The study will be performed in accordance with the recommendations guiding research involving human subjects adopted by the 18th World Medical Association General Assembly, Helsinki, Finland, June 1964, amended at the 64th World Medical Association General Assembly, Fortaleza, Brazil, October 2013. The study will be reported in accordance with the STROBE. ${ }^{33}$ The results will be presented in scientific conferences and published in peer-reviewed academic journals as well as being disseminated to the Swedish football movement through stakeholders and media.

\section{Author affiliations}

${ }^{1}$ Swedish School of Sport and Health Sciences, Stockholm, Sweden

${ }^{2}$ Institute of Environmental Medicine, Karolinska Institutet, Stockholm, Sweden
${ }^{3}$ Department of Health Promotion Science, Sophiahemmet University, Stockholm, Sweden

${ }^{4}$ Department of Health, Medicine and Caring Sciences, Linköping University, Linkoping, Sweden

${ }^{5}$ Sport Without Injury ProgrammE (SWIPE), Linköping University, Linkoping, Sweden ${ }^{6}$ Football Research Group, Linköping, Sweden

${ }^{7}$ Department of Orthopaedics, Hässleholm-Kristianstad Hospitals, Hässleholm, Sweden

${ }^{8}$ Unit of Community Medicine, Department of Health, Medicine and Caring Sciences, Linköping University, Linköping, Sweden

${ }^{9}$ Halmstad University, Halmstad, Sweden

${ }^{10}$ Scandinavian College of Naprapathic Manual Medicine, Stockholm, Sweden

Twitter Ulrika Tranaeus @UlrikaTranaeus, Nathan Weiss @nathnweiss, Martin Hagglund @MHgglund, Markus Waldén @MarkusWalden and Martin Asker@ martinasker

Acknowledgements Martin Samuelsson, Lena Holm and Henrik Källberg have been involved in planning of study design, Joakim Bogren, Victor Ramirez Kristiansen, contributed to the first version of the draft. Students from the classes DN 47, 48, 49, 50, 51 and 53 at the Scandinavian college of Naprapathic Manual Medicine, Stockholm, Sweden are participating during inclusion of participants, the physical tests and the prospective data collection.

Contributors VL, ES, MA and UT initiated the study. All authors, UT, NW, VL, MH, MW, UJ and ES conceived the study and contributed to the development of the study protocol. ES is the study guarantor. UT and NW bi-drafted the manuscript which was critically revised in steps by all coauthors. The final manuscript was approved by all authors.

Funding This study is funded by grants from Swedish Research Council for Sport Science (grant number: P2019-0045, P2020-0100). The Swedish Naprapathic, and the Norwegian Naprapathic Associations, Active life foundation and Sophiahemmet foundation. There are no grant numbers for the latter funders (award/grant number: $\mathrm{N} / \mathrm{A})$.

Competing interests None declared.

Patient consent for publication Not applicable.

Provenance and peer review Not commissioned; externally peer reviewed.

Supplemental material This content has been supplied by the author(s). It has not been vetted by BMJ Publishing Group Limited (BMJ) and may not have been peer-reviewed. Any opinions or recommendations discussed are solely those of the author(s) and are not endorsed by BMJ. BMJ disclaims all liability and responsibility arising from any reliance placed on the content. Where the content includes any translated material, BMJ does not warrant the accuracy and reliability of the translations (including but not limited to local regulations, clinical guidelines, terminology, drug names and drug dosages), and is not responsible for any error and/or omissions arising from translation and adaptation or otherwise.

Open access This is an open access article distributed in accordance with the Creative Commons Attribution 4.0 Unported (CC BY 4.0) license, which permits others to copy, redistribute, remix, transform and build upon this work for any purpose, provided the original work is properly cited, a link to the licence is given, and indication of whether changes were made. See: https://creativecommons.org/ licenses/by/4.0/.

\section{ORCID iDs}

Ulrika Tranaeus http://orcid.org/0000-0002-2102-6352

Nathan Weiss http://orcid.org/0000-0002-1814-020X

Martin Hagglund http://orcid.org/0000-0002-6883-1471

Markus Waldén http://orcid.org/0000-0002-6790-4042

Urban Johnson http://orcid.org/0000-0003-0990-4842

Martin Asker http://orcid.org/0000-0002-5387-3572

Eva Skillgate http://orcid.org/0000-0003-2096-1530

\section{REFERENCES}

1 Federation Internationale de Football Association. Women's football survey, 2014.

2 López-Valenciano A, Ruiz-Pérez I, Garcia-Gómez A, et al. Epidemiology of injuries in professional football: a systematic review and meta-analysis. Br J Sports Med 2020;54:711-8. 
3 Bennett P, Fawcett L. Trauma injuries sustained by female footballers. Trauma 2006;8:69-76.

4 Hägglund M, Waldén M, Ekstrand J. Injuries among male and female elite football players. Scand J Med Sci Sports 2009;19:819-27.

5 Faude O, Junge A, Kindermann W, et al. Injuries in female soccer players: a prospective study in the German national League. Am J Sports Med 2005;33:1694-700.

6 Le Gall F, Carling C, Reilly T. Injuries in young elite female soccer players. Am J Sports Med 2008;36:276-84.

7 Clausen MB, Zebis MK, Møller M, et al. High injury incidence in adolescent female soccer. Am J Sports Med 2014;42:2487-94.

8 Larruskain J, Lekue JA, Diaz N, et al. A comparison of injuries in elite male and female football players: a five-season prospective study. Scand J Med Sci Sports 2018;28:237-45.

9 Lohmander LS, Ostenberg A, Englund M, et al. High prevalence of knee osteoarthritis, pain, and functional limitations in female soccer players twelve years after anterior cruciate ligament injury. Arthritis Rheum 2004;50:3145-52.

10 Prien A, Prinz B, Dvořák J, et al. Health problems in former elite female football players: prevalence and risk factors. Scand J Med Sci Sports 2017;27:1404-10.

11 Roos EM. Joint injury causes knee osteoarthritis in young adults. Curr Opin Rheumatol 2005;17:195-200.

12 Wylleman P, Reints A. A lifespan perspective on the career of talented and elite athletes: perspectives on high-intensity sports. Scand J Med Sci Sports 2010;20:88-94.

13 Putukian M. The psychological response to injury in student athletes: a narrative review with a focus on mental health. $\mathrm{Br} J$ Sports Med 2016;50:145-8

14 van Mechelen W, Hlobil H, Kemper HC. Incidence, severity, aetiology and prevention of sports injuries. A review of concepts. Sports Med 1992;14:82-99.

15 Dvorak J, Junge A, Chomiak J, et al. Risk factor analysis for injuries in football players - Possibilities for a prevention program. Am J Sports Med 2000;28:S69-74.

16 Steffen K, Engebretsen L. More data needed on injury risk among young elite athletes. Br J Sports Med 2010;44:485-9.

17 Wiese-Bjornstal DM. Psychology and socioculture affect injury risk, response, and recovery in high-intensity athletes: a consensus statement. Scand J Med Sci Sports 2010;20:103-11.

18 Hägglund M, Waldén M. Risk factors for acute knee injury in female youth football. Knee Surg Sports Traumatol Arthrosc 2016;24:737-46.

19 Faude O, Junge A, Kindermann W, et al. Risk factors for injuries in elite female soccer players. Br J Sports Med 2006;40:785-90.

20 Steffen K, Pensgaard AM, Bahr R. Self-reported psychological characteristics as risk factors for injuries in female youth football. Scand J Med Sci Sports 2009;19:442-51.

21 Alahmad TA, Kearney P, Cahalan R. Injury in elite women's soccer: a systematic review. Phys Sportsmed 2020:1-7.

22 Magnusson K, Turkiewicz A, Hughes V, et al. High genetic contribution to anterior cruciate ligament rupture: heritability $\sim 69 . \mathrm{Br}$ J Sports Med 2020;55:385-9.

23 Ostenberg A, Roos H. Injury risk factors in female European football. A prospective study of 123 players during one season. Scand J Med Sci Sports 2000;10:279-85.

24 Watson A, Brickson S, Brooks MA, et al. Preseason aerobic fitness predicts in-season injury and illness in female youth athletes. Orthop J Sports Med 2017;5:232596711772697.

25 Söderman K, Alfredson H, Pietilä T, et al. Risk factors for leg injuries in female soccer players: a prospective investigation during one out-door season. Knee Surg Sports Traumatol Arthrosc 2001;9:313-21.

26 Nilstad A, Andersen TE, Bahr R, et al. Risk factors for lower extremity injuries in elite female soccer players. Am J Sports Med 2014;42:940-8

27 Blokland D, Thijs KM, Backx FJG, et al. No effect of generalized joint hypermobility on injury risk in elite female soccer players: a prospective cohort study. Am J Sports Med 2017;45:286-93.

28 Ehrmann Feldman D, Shrier I, Rossignol M, et al. Risk factors for the development of neck and upper limb pain in adolescents. Spine 2002;27:523-8.

29 Johnson U, Ivarsson A. Psychological predictors of sport injuries among junior soccer players. Scand J Med Sci Sports 2011;21:129-36.

30 Ivarsson A, Johnson U, Lindwall M, et al. Psychosocial stress as a predictor of injury in elite junior soccer: a latent growth curve analysis. J Sci Med Sport 2014;17:366-70.

31 Junge A, Rösch D, Peterson L, et al. Prevention of soccer injuries: a prospective intervention study in youth amateur players. Am J Sports Med 2002;30:652-9.
32 Pensgaard AM, Ivarsson A, Nilstad A, et al. Psychosocial stress factors, including the relationship with the coach, and their influence on acute and overuse injury risk in elite female football players. BMJ Open Sport Exerc Med 2018;4:e000317.

33 von Elm E, Altman DG, Egger M, et al. The strengthening the reporting of observational studies in epidemiology (STROBE) statement: guidelines for reporting observational studies. Int J Surg 2014;12:1495-9.

34 Waldén M, Atroshi I, Magnusson $\mathrm{H}$, et al. Prevention of acute knee injuries in adolescent female football players: cluster randomised controlled trial. BMJ 2012;344:e3042.

35 Fuller CW, Ekstrand J, Junge A. Consensus statement on injury definitions and data collection procedures in studies of football (soccer) injuries. Br J Sports Med 2006;40:193-201.

36 Hägglund M, Waldén M, Bahr R, et al. Methods for epidemiological study of injuries to professional football players: developing the UEFA model. Br J Sports Med 2005;39:340-6.

37 Emery CA, Meeuwisse WH, Hartmann SE. Evaluation of risk factors for injury in adolescent soccer: implementation and validation of an injury surveillance system. Am J Sports Med 2005;33:1882-91.

38 Hardy GE, Shapiro DA, Haynes CE, et al. Validation of the General Health Questionnaire-12: using a sample of employees from England's health care services. Psychol Assess 1999;11:159-65.

39 Carver CS. You want to measure coping but your protocol's too long: consider the Brief cope. Int J Behav Med 1997;4:92-100.

40 Vallerand RJ, Blanchard C, Mageau GA, et al. Les passions de I'ame: on obsessive and harmonious passion. J Pers Soc Psychol 2003;85:756-67.

41 Salminen S, Kouvonen A, Koskinen A, et al. Is a single item stress measure independently associated with subsequent severe injury: a prospective cohort study of 16,385 forest industry employees. BMC Public Health 2014;14:543.

42 Clarsen B, Myklebust G, Bahr R. Development and validation of a new method for the registration of overuse injuries in sports injury epidemiology: the Oslo Sports Trauma Research Centre (OSTRC) overuse injury questionnaire. Br J Sports Med 2013;47:495-502.

43 Ekman E, Frohm A, Ek P, et al. Swedish translation and validation of a web-based questionnaire for registration of overuse problems. Scand J Med Sci Sports 2015;25:104-9.

44 Von Korff M, Ormel J, Keefe FJ, et al. Grading the severity of chronic pain. Pain 1992;50:133-49.

45 Dunn KM, Campbell P, Jordan KP. Validity of the visual trajectories questionnaire for pain. J Pain 2017;18:1451-8.

46 Dennis RJ, Finch CF, Elliott BC, et al. The reliability of musculoskeletal screening tests used in cricket. Phys Ther Sport 2008:9:25-33.

47 Youdas JW, Bogard CL, Suman VJ. Reliability of goniometric measurements and visual estimates of ankle joint active range of motion obtained in a clinical setting. Arch Phys Med Rehabil 1993;74:1113-8

48 Ness BM, Sudhagoni RG, Tao H, et al. The reliability of a novel heelrise test versus goniometry to assess plantarflexion range of motion. Int J Sports Phys Ther 2018;13:19-27.

49 Konor MM, Morton S, Eckerson JM, et al. Reliability of three measures of ankle dorsiflexion range of motion. Int J Sports Phys Ther 2012;7:279.

50 Frohm A, Heijne A, Kowalski J, et al. A nine-test screening battery for athletes: a reliability study. Scand J Med Sci Sports 2012;22:306-15.

51 Asker M, Waldén M, Källberg $\mathrm{H}$, et al. A prospective cohort study identifying risk factors for shoulder injuries in adolescent elite handball players: the Karolinska Handball Study (KHAST) study protocol. BMC Musculoskelet Disord 2017:18:485.

52 Johnson KD, Kim K-M, Yu B-K, et al. Reliability of thoracic spine rotation range-of-motion measurements in healthy adults. J Athl Train 2012;47:52-60.

53 Andre MJ, Fry AC, Heyrman MA, et al. A reliable method for assessing rotational power. J Strength Cond Res 2012;26:720-4

54 Palmer TG, Uhl TL. interday reliability of peak muscular power outputs on an isotonic dynamometer and assessment of active trunk control using the CHOP and lift tests. J Athl Train 2011;46:150-9.

55 Zois J, P Sharp A, Talukdar K, et al. The reliability of a rotational power assessment of the core. J Athl Enhanc 2016;5.

56 Arumugam A, Mani R, Raja K. Interrater reliability of the craniocervical flexion test in asymptomatic individuals--a crosssectional study. J Manipulative Physiol Ther 2011;34:247-53.

57 James G, Doe T. The craniocervical flexion test: intra-tester reliability in asymptomatic subjects. Physiother Res Int 2010;15:144-9.

58 Thorborg K, Bandholm T, Hölmich P. Hip- and knee-strength assessments using a hand-held dynamometer with external beltfixation are inter-tester reliable. Knee Surg Sports Traumatol Arthrosc 2013;21:550-5 
59 Kelln BM, McKeon PO, Gontkof LM, et al. Hand-held dynamometry: reliability of lower extremity muscle testing in healthy, physically active,young adults. J Sport Rehabil 2008;17:160-70.

60 Prather H, Harris-Hayes M, Hunt DM, et al. Reliability and agreement of hip range of motion and provocative physical examination tests in asymptomatic volunteers. PM R 2010;2:888-95.

61 Nussbaumer S, Leunig M, Glatthorn JF, et al. Validity and testretest reliability of manual goniometers for measuring passive hip range of motion in femoroacetabular impingement patients. $B M C$ Musculoskelet Disord 2010;11:194.

62 Sharma N, Sharma A, Singh Sandhu J. Functional performance testing in athletes with functional ankle instability. Asian J Sports Med 2011;2:249.

63 Caffrey E, Docherty CL, Schrader J, et al. The ability of 4 singlelimb hopping tests to detect functional performance deficits in individuals with functional ankle instability. J Orthop Sports Phys Ther 2009;39:799-806.

64 Vaseenon T, Gao Y, Phisitkul P. Comparison of two manual tests for ankle laxity due to rupture of the lateral ankle ligaments. lowa Orthop J 2012;32:9-16.
65 de Vries JS, Kerkhoffs GMMJ, Blankevoort L, et al. Clinical evaluation of a dynamic test for lateral ankle ligament laxity. Knee Surg Sports Traumatol Arthrosc 2010;18:628-33.

66 Smith TO, Clark A, Neda S, et al. The intra- and inter-observer reliability of the physical examination methods used to assess patients with patellofemoral joint instability. Knee 2012;19:404-10.

67 Moreau CE, Green BN, Johnson CD, et al. Isometric back extension endurance tests: a review of the literature. J Manipulative Physiol Ther 2001;24:110-22.

68 Demoulin C, Vanderthommen M, Duysens C, et al. Spinal muscle evaluation using the Sorensen test: a critical appraisal of the literature. Joint Bone Spine 2006;73:43-50.

69 Latimer J, Maher CG, Refshauge K, et al. The reliability and validity of the Biering-Sorensen test in asymptomatic subjects and subjects reporting current or previous nonspecific low back pain. Spine 1999;24:2085-9.

70 Malone S, Owen A, Newton M, et al. The acute:chonic workload ratio in relation to injury risk in professional soccer. J Sci Med Sport 2017;20:561-5

71 Nilstad A, Bahr R, Andersen TE. Text messaging as a new method for injury registration in sports: a methodological study in elite female football. Scand J Med Sci Sports 2014;24:243-9. 